\title{
The problem with fuzzy eigenvalue parameter in one of the boundary conditions
}

\author{
Hülya Gültekin Çitil* \\ Department of Mathematics, Faculty of Arts and Sciences, Giresun University, Turkey \\ hulya.citil@giresun.edu.tr
}

\section{ARTICLE INFO}

\section{Article History:}

Received 15 March 2020

Accepted 26 April 2020

Available 31 May 2020

Keywords:

Sturm-Liouville fuzzy problem

Fuzzy eigenvalue

Fuzzy eigenfunction

\section{ABSTRACT}

In this work, we study the problem with fuzzy eigenvalue parameter in one of the boundary conditions. We find fuzzy eigenvalues of the problem using the Wronskian functions $\underline{W}_{\alpha}(\lambda)$ and $\bar{W}_{\alpha}(\lambda)$. Also, we find eigenfunctions associated with eigenvalues. We draw graphics of eigenfunctions.

\section{Introduction}

Fuzzy logic is studied in many areas [1,2]. To solve many problems, Sturm-Liouville Theory is used in mathematical physics 3, 4. Sturm-Liouville fuzzy problem was defined by Gültekin Citil and Altınışı [5]. They studied Sturm-Liouville fuzzy problems with reel and fuzzy coefficients in the boundary conditions under the Hukuhara differentiability [6,7]. Also, fuzzy eigenvalue problems were investigated under the approach of generalized differentiability in many papers 8,9 . In the other hand, the fuzzy problem with eigenvalue parameter in the boundary condition was studied 10,11. But, eigenvalue parameter was not fuzzy in these papers. The problem with fuzzy eigenvalue parameter was defined and investigated by Gültekin Çitil [12.

This paper is on the problem with fuzzy eigenvalue parameter in one of the boundary conditions. That is, we concern the fuzzy eigenvalue problem

$$
\tau=\frac{d^{2}}{d t^{2}},
$$

$$
\begin{gathered}
\tau u+[\lambda]^{\alpha} u=0, t \in(a, b) \\
{[A]^{\alpha} u(a)+[\lambda]^{\alpha}[B]^{\alpha} u^{\prime}(a)=0,} \\
{[C]^{\alpha} u(b)+[D]^{\alpha} u^{\prime}(b)=0,}
\end{gathered}
$$

where $[A]^{\alpha}=\left[\underline{A}_{\alpha}, \bar{A}_{\alpha}\right],[C]^{\alpha}=\left[\underline{C}_{\alpha}, \bar{C}_{\alpha}\right]$ are negative triangular fuzzy numbers, $[B]^{\alpha}=\left[\underline{B}_{\alpha}, \bar{B}_{\alpha}\right]$, $[D]^{\alpha}=\left[\underline{D}_{\alpha}, \bar{D}_{\alpha}\right]$ are positive triangular fuzzy numbers, $[\lambda]^{\alpha}=\left[\underline{\lambda}_{\alpha}, \bar{\lambda}_{\alpha}\right]$ is positive fuzzy eigenvalue parameter and $u(t, \lambda)$ is positive fuzzy function.

Definition 1. [13] A fuzzy number is a mapping $u: \mathbb{R} \rightarrow[0,1]$ satisfying the following properties: $u$ is normal,

$u$ is convex fuzzy set, $u$ is upper semi-continuous on $\mathbb{R}$, $c l\{x \in \mathbb{R} \mid u(x)>0\}$ is compact, where cl denotes the closure of a subset.

We show the space of fuzzy sets with $\mathbb{R}_{F}$.

Definition 2. [14] Let $u \in \mathbb{R}_{F}$. The $\alpha$-level set of $u$ is defined as

$$
[u]^{\alpha}=\{x \in \mathbb{R} \mid u(x) \geq \alpha\}, 0<\alpha \leq 1
$$

*Corresponding Author 
The $\alpha$-level set of $u$ is denoted as

$$
[u]^{\alpha}=\left[\underline{u}_{\alpha}, \bar{u}_{\alpha}\right] .
$$

Definition 3. [15]A fuzzy number $u$ is called positive (negative), denoted by $u>0(u<0)$, if its membership function $u(x)$ satisfies $u(x)=0$, $\forall x<0(x>0)$.

Remark 1. [14] The sufficient and necessary conditions for $\left[\underline{u}_{\alpha}, \bar{u}_{\alpha}\right]$ to define the parametric form of a fuzzy number as follows:

$\underline{u}_{\alpha}$ is bounded monotonic increasing (nondecreasing) left-continuous function on $(0,1]$ and rightcontinuous for $\alpha=0$,

$\bar{u}_{\alpha}$ is bounded monotonic decreasing (nonincreasing) left-continuous function on $(0,1]$ and rightcontinuous for $\alpha=0$,

$\underline{u}_{\alpha} \leq \bar{u}_{\alpha}, 0 \leq \alpha \leq 1$.

Definition 4. 14 For $u, v \in \mathbb{R}_{F}$ and $\lambda \in \mathbb{R}$, the sum $u+v$ and the product $\lambda u$ are defined by $[u+v]^{\alpha}=[u]^{\alpha}+[v]^{\alpha},[\lambda u]^{\alpha}=\lambda[u]^{\alpha}$ where means the usual addition of two intervals (subsets) of $\mathbb{R}$ and $\lambda[u]^{\alpha}$ means the usual product between a scalar and a subset of $\mathbb{R}$.

Definition 5. [16] Let $u, v \in \mathbb{R}_{F},[u]^{\alpha}=$ $\left[\underline{u}_{\alpha}, \bar{u}_{\alpha}\right],[v]^{\alpha}=\left[\underline{v}_{\alpha}, \bar{v}_{\alpha}\right]$. The product uv is defined by

$$
[u v]^{\alpha}=[u]^{\alpha}[v]^{\alpha}, \forall \alpha \in[0,1],
$$

where

$$
\begin{gathered}
{[u]^{\alpha}[v]^{\alpha}=\left[\underline{u}_{\alpha}, \bar{u}_{\alpha}\right]\left[\underline{v}_{\alpha}, \bar{v}_{\alpha}\right]=\left[\underline{w}_{\alpha}, \bar{w}_{\alpha}\right],} \\
\underline{w}_{\alpha}=\min \left\{\underline{u}_{\alpha} \underline{v}_{\alpha}, \underline{u}_{\alpha} \bar{v}_{\alpha}, \bar{u}_{\alpha} \underline{v}_{\alpha}, \bar{u}_{\alpha} \bar{v}_{\alpha}\right\}, \\
\bar{w}_{\alpha}=\max \left\{\underline{u}_{\alpha} \underline{v}_{\alpha}, \underline{u}_{\alpha} \bar{v}_{\alpha}, \bar{u}_{\alpha} \underline{v}_{\alpha}, \bar{u}_{\alpha} \bar{v}_{\alpha}\right\} .
\end{gathered}
$$

Definition 6. [17] Let $u, v \in \mathbb{R}_{F}$. If there exists $w \in \mathbb{R}_{F}$ such that $u=v+w$, then $w$ is called the Hukuhara difference of fuzzy numbers $u$ and $v$, and it is denoted by $w=u \ominus v$.

Definition 7. [14, 18] Let $f:[a, b] \rightarrow \mathbb{R}_{F}$ and $t_{0} \in[a, b]$. We say that $f$ is Hukuhara differentiable at $t_{0}$, if there exists an element $f^{\prime}\left(t_{0}\right) \in$ $\mathbb{R}_{F}$ such that for all $h>0$ sufficiently small, $\exists f\left(t_{0}+h\right) \ominus f\left(t_{0}\right), f\left(t_{0}\right) \ominus f\left(t_{0}-h\right)$ and the limits hold

$$
\begin{aligned}
\lim _{h \rightarrow 0} \frac{f\left(t_{0}+h\right) \ominus f\left(t_{0}\right)}{h} & =\lim _{h \rightarrow 0} \frac{f\left(t_{0}\right) \ominus f\left(t_{0}-h\right)}{h} \\
& =f^{\prime}\left(t_{0}\right) .
\end{aligned}
$$

\section{The fuzzy eigenvalues and fuzzy eigenfunctions of the problem}

In this section, we investigate the fuzzy eigenvalues and the fuzzy eigenfunctions of the problem (11)-(33) .

Let be $[\lambda]^{\alpha}=\left[\underline{\lambda}_{\alpha}, \bar{\lambda}_{\alpha}\right]=\left[\underline{k}_{\alpha}^{2}, \bar{k}_{\alpha}^{2}\right], \underline{k}_{\alpha}>0$, $\bar{k}_{\alpha}>0$. Then, using the Hukuhara differentiability and fuzzy arithmetic, the general solution of the fuzzy differential equation (11) is

$\underline{u}_{\alpha}(t, \lambda)=c_{1}(\alpha, \lambda) \cos \left(\underline{k}_{\alpha} t\right)+c_{2}(\alpha, \lambda) \sin \left(\underline{k}_{\alpha} t\right)$,

$\bar{u}_{\alpha}(t, \lambda)=c_{3}(\alpha, \lambda) \cos \left(\bar{k}_{\alpha} t\right)+c_{4}(\alpha, \lambda) \sin \left(\bar{k}_{\alpha} t\right)$,

$$
[u(t, \lambda)]^{\alpha}=\left[\underline{u}_{\alpha}(t, \lambda), \bar{u}_{\alpha}(t, \lambda)\right] .
$$

Let

$$
[\varphi(t, \lambda)]^{\alpha}=\left[\underline{\varphi}_{\alpha}(t, \lambda), \bar{\varphi}_{\alpha}(t, \lambda)\right]
$$

be the solution of the equation (11) satisfying the conditions

$$
u(a)=[\lambda]^{\alpha}[B]^{\alpha}, u^{\prime}(a)=-[A]^{\alpha}
$$

and

$$
[\chi(t, \lambda)]^{\alpha}=\left[\underline{\chi}_{\alpha}(t, \lambda), \bar{\chi}_{\alpha}(t, \lambda)\right]
$$

be the solution of the equation (1) satisfying the conditions

$$
u(b)=[D]^{\alpha}, u^{\prime}(b)=-[C]^{\alpha}
$$

Then, $\underline{\varphi}_{\alpha}(t, \lambda), \bar{\varphi}_{\alpha}(t, \lambda), \underline{\chi}_{\alpha}(t, \lambda), \bar{\chi}_{\alpha}(t, \lambda)$ can be shown as

$\underline{\varphi}_{\alpha}(t, \lambda)=c_{11}(\alpha, \lambda) \cos \left(\underline{k}_{\alpha} t\right)+c_{21}(\alpha, \lambda) \sin \left(\underline{k}_{\alpha} t\right)$,

$\bar{\varphi}_{\alpha}(t, \lambda)=c_{31}(\alpha, \lambda) \cos \left(\bar{k}_{\alpha} t\right)+c_{41}(\alpha, \lambda) \sin \left(\bar{k}_{\alpha} t\right)$,

$\underline{\chi}_{\alpha}(t, \lambda)=c_{12}(\alpha, \lambda) \cos \left(\underline{k}_{\alpha} t\right)+c_{22}(\alpha, \lambda) \sin \left(\underline{k}_{\alpha} t\right)$,

$\bar{\chi}_{\alpha}(t, \lambda)=c_{32}(\alpha, \lambda) \cos \left(\bar{k}_{\alpha} t\right)+c_{42}(\alpha, \lambda) \sin \left(\bar{k}_{\alpha} t\right)$.

For $[\varphi(t, \lambda)]^{\alpha}$, from the first condition in (7), since $[B]^{\alpha}=\left[\underline{B}_{\alpha}, \bar{B}_{\alpha}\right]$ is positive fuzzy number, we have

$$
[\lambda]^{\alpha}[B]^{\alpha}=\left[\underline{k}_{\alpha}^{2}, \bar{k}_{\alpha}^{2}\right]\left[\underline{B}_{\alpha}, \bar{B}_{\alpha}\right]=\left[\underline{k}_{\alpha}^{2} \underline{B}_{\alpha}, \bar{k}_{\alpha}^{2} \bar{B}_{\alpha}\right] .
$$


Then, using the conditions (77), it is obtained

$c_{11}(\alpha, \lambda) \cos \left(\underline{k}_{\alpha} a\right)+c_{21}(\alpha, \lambda) \sin \left(\underline{k}_{\alpha} a\right)=\underline{k}_{\alpha}^{2} \underline{B}_{\alpha}$,

$$
c_{12}(\alpha, \lambda) \cos \left(\underline{k}_{\alpha} b\right)+c_{22}(\alpha, \lambda) \sin \left(\underline{k}_{\alpha} b\right)=\underline{D}_{\alpha}
$$

$c_{11}(\alpha, \lambda) \underline{k}_{\alpha} \sin \left(\underline{k}_{\alpha} a\right)-c_{21}(\alpha, \lambda) \underline{k}_{\alpha} \cos \left(\underline{k}_{\alpha} a\right)=\bar{A}_{\alpha}$,

$c_{12}(\alpha, \lambda) \underline{k}_{\alpha} \sin \left(\underline{k}_{\alpha} b\right)-c_{22}(\alpha, \lambda) \underline{k}_{\alpha} \cos \left(\underline{k}_{\alpha} b\right)=\bar{C}_{\alpha}$,

$c_{31}(\alpha, \lambda) \cos \left(\bar{k}_{\alpha} a\right)+c_{41}(\alpha, \lambda) \sin \left(\bar{k}_{\alpha} a\right)=\bar{k}_{\alpha}^{2} \bar{B}_{\alpha}$,

$$
c_{32}(\alpha, \lambda) \cos \left(\bar{k}_{\alpha} b\right)+c_{42}(\alpha, \lambda) \sin \left(\bar{k}_{\alpha} b\right)=\bar{D}_{\alpha},
$$

$c_{31}(\alpha, \lambda) \bar{k}_{\alpha} \sin \left(\bar{k}_{\alpha} a\right)-c_{41}(\alpha, \lambda) \bar{k}_{\alpha} \cos \left(\bar{k}_{\alpha} a\right)=\underline{A}_{\alpha}$.

$c_{32}(\alpha, \lambda) \bar{k}_{\alpha} \sin \left(\bar{k}_{\alpha} b\right)-c_{42}(\alpha, \lambda) \bar{k}_{\alpha} \cos \left(\bar{k}_{\alpha} b\right)=\underline{C}_{\alpha}$.

From (9)-(10),

From (13)-(14),

$$
\begin{aligned}
& c_{11}(\alpha, \lambda)=\frac{\underline{k}_{\alpha}^{3} \underline{B}_{\alpha} \cos \left(\underline{k}_{\alpha} a\right)+\bar{A}_{\alpha} \sin \left(\underline{k}_{\alpha} a\right)}{\underline{k}_{\alpha}}, \\
& c_{21}(\alpha, \lambda)=\frac{\underline{k}_{\alpha}^{3} \underline{B}_{\alpha} \sin \left(\underline{k}_{\alpha} a\right)-\bar{A}_{\alpha} \cos \left(\underline{k}_{\alpha} a\right)}{\underline{k}_{\alpha}}
\end{aligned}
$$

are obtained. From (11)-(12), we have

$$
\begin{aligned}
c_{31}(\alpha, \lambda) & =\frac{\bar{k}_{\alpha}^{3} \bar{B}_{\alpha} \cos \left(\bar{k}_{\alpha} a\right)+\underline{A}_{\alpha} \sin \left(\bar{k}_{\alpha} a\right)}{\bar{k}_{\alpha}}, \\
c_{41}(\alpha, \lambda) & =\frac{\bar{k}_{\alpha}^{3} \bar{B}_{\alpha} \sin \left(\bar{k}_{\alpha} a\right)-\underline{A}_{\alpha} \sin \left(\bar{k}_{\alpha} a\right)}{\bar{k}_{\alpha}} .
\end{aligned}
$$

$$
\begin{aligned}
& c_{12}(\alpha, \lambda)=\frac{\underline{D}_{\alpha} \cos \left(\underline{k}_{\alpha} b\right)+\bar{C}_{\alpha} \sin \left(\underline{k}_{\alpha} b\right)}{\underline{k}_{\alpha}}, \\
& c_{22}(\alpha, \lambda)=\frac{\underline{D}_{\alpha} \sin \left(\underline{k}_{\alpha} b\right)-\bar{C}_{\alpha} \cos \left(\underline{k}_{\alpha} b\right)}{\underline{k}_{\alpha}}
\end{aligned}
$$

are obtained. From (15)-(16), we have

$$
\begin{aligned}
c_{32}(\alpha, \lambda) & =\frac{\bar{D}_{\alpha} \cos \left(\bar{k}_{\alpha} b\right)+\underline{C}_{\alpha} \sin \left(\bar{k}_{\alpha} b\right)}{\bar{k}_{\alpha}}, \\
c_{42}(\alpha, \lambda) & =\frac{\bar{D}_{\alpha} \sin \left(\bar{k}_{\alpha} b\right)-\underline{C}_{\alpha} \sin \left(\bar{k}_{\alpha} b\right)}{\bar{k}_{\alpha}} .
\end{aligned}
$$

Then, the solution of the equation (11) satisfying the conditions (7) is

Then, solution of the equation (1D) satisfying the conditions (8) is

$$
\begin{aligned}
\underline{\varphi}_{\alpha}(t, \lambda)= & \left(\underline{k}_{\alpha}^{2} \underline{B}_{\alpha} \cos \left(\underline{k}_{\alpha} a\right)\right. \\
& \left.+\frac{\bar{A}_{\alpha}}{\underline{k}_{\alpha}} \sin \left(\underline{k}_{\alpha} a\right)\right) \cos \left(\underline{k}_{\alpha} t\right) \\
& +\left(\underline{k}_{\alpha}^{2} \underline{B}_{\alpha} \sin \left(\underline{k}_{\alpha} a\right)\right. \\
& \left.-\frac{\bar{A}_{\alpha}}{\underline{k}_{\alpha}} \cos \left(\underline{k}_{\alpha} a\right)\right) \sin \left(\underline{k}_{\alpha} t\right), \\
\bar{\varphi}_{\alpha}(t, \lambda)= & \left(\bar{k}_{\alpha}^{2} \bar{B}_{\alpha} \cos \left(\bar{k}_{\alpha} a\right)\right. \\
& \left.\underline{A}_{\alpha} \sin \left(\bar{k}_{\alpha} a\right)\right) \cos \left(\bar{k}_{\alpha} t\right) \\
& +\left(\bar{k}_{\alpha}^{2} \bar{B}_{\alpha} \sin \left(\bar{k}_{\alpha} a\right)\right. \\
& \left.-\frac{\underline{A}_{\alpha}}{\bar{k}_{\alpha}} \cos \left(\bar{k}_{\alpha} a\right)\right) \sin \left(\bar{k}_{\alpha} t\right), \\
{[\varphi(t, \lambda)]^{\alpha}=} & {\left[\underline{\varphi}_{\alpha}(t, \lambda), \bar{\varphi}_{\alpha}(t, \lambda)\right] . }
\end{aligned}
$$

$$
\begin{aligned}
& \underline{\chi}_{\alpha}(t, \lambda)=\left(\frac{\underline{D}_{\alpha}}{\underline{k}_{\alpha}} \cos \left(\underline{k}_{\alpha} b\right)\right. \\
& \left.+\frac{\bar{C}_{\alpha}}{\underline{k}_{\alpha}} \sin \left(\underline{k}_{\alpha} b\right)\right) \cos \left(\underline{k}_{\alpha} t\right) \\
& +\left(\frac{\underline{D}_{\alpha}}{\underline{k}_{\alpha}} \sin \left(\underline{k}_{\alpha} b\right)\right. \\
& \left.-\frac{\bar{C}_{\alpha}}{\underline{k}_{\alpha}} \cos \left(\underline{k}_{\alpha} b\right)\right) \sin \left(\underline{k}_{\alpha} t\right), \\
& \bar{\chi}_{\alpha}(t, \lambda)=\left(\frac{\bar{D}_{\alpha}}{\bar{k}_{\alpha}} \cos \left(\bar{k}_{\alpha} b\right)\right. \\
& \left.+\frac{C_{\alpha}}{\bar{k}_{\alpha}} \sin \left(\bar{k}_{\alpha} b\right)\right) \cos \left(\bar{k}_{\alpha} t\right) \\
& +\left(\frac{\bar{D}_{\alpha}}{\bar{k}_{\alpha}} \sin \left(\bar{k}_{\alpha} b\right)\right. \\
& \begin{aligned}
& \left.-\frac{C_{\alpha}}{\bar{k}_{\alpha}} \cos \left(\bar{k}_{\alpha} b\right)\right) \sin \left(\bar{k}_{\alpha} t\right), \\
{[\chi(t, \lambda)]^{\alpha} } & =\left[\underline{\chi}_{\alpha}(t, \lambda), \bar{\chi}_{\alpha}(t, \lambda)\right] .
\end{aligned} \\
& \begin{aligned}
& \left.-\frac{C_{\alpha}}{\bar{k}_{\alpha}} \cos \left(\bar{k}_{\alpha} b\right)\right) \sin \left(\bar{k}_{\alpha} t\right), \\
{[\chi(t, \lambda)]^{\alpha} } & =\left[\underline{\chi}_{\alpha}(t, \lambda), \bar{\chi}_{\alpha}(t, \lambda)\right] .
\end{aligned}
\end{aligned}
$$

For $[\chi(t, \lambda)]^{\alpha}$, using the conditions (8), we have the equations 
Since the eigenvalues of the fuzzy boundary value problem (11)- (3) if and only if are consist of the zeros of functions $W\left(\underline{\varphi}_{\alpha}, \underline{\chi}_{\alpha}\right)(t, \lambda)$ and $W\left(\bar{\varphi}_{\alpha}, \bar{\chi}_{\alpha}\right)(t, \lambda)[5$, we find Wronskian functions

$$
\begin{aligned}
W\left(\underline{\varphi}_{\alpha}, \underline{\chi}_{\alpha}\right)(t, \lambda)= & \underline{\varphi}_{\alpha}(t, \lambda) \underline{\chi}_{\alpha}^{\prime}(t, \lambda) \\
& -\underline{\chi}_{\alpha}(t, \lambda) \underline{\varphi}_{\alpha}^{\prime}(t, \lambda), \\
W\left(\bar{\varphi}_{\alpha}, \bar{\chi}_{\alpha}\right)(t, \lambda)= & \bar{\varphi}_{\alpha}(t, \lambda) \bar{\chi}_{\alpha}^{\prime}(t, \lambda) \\
& -\bar{\chi}_{\alpha}(t, \lambda) \bar{\varphi}_{\alpha}^{\prime}(t, \lambda) .
\end{aligned}
$$

Computing the values (17) and (18) and making the necessary operations, we obtain

$$
\begin{aligned}
W\left(\underline{\varphi}_{\alpha}, \underline{\chi}_{\alpha}\right)(\lambda)= & \left(\frac{\bar{A}_{\alpha} \underline{D}_{\alpha}}{\underline{k}_{\alpha}}\right. \\
& \left.-\underline{k}_{\alpha}^{2} \underline{B}_{\alpha} \bar{C}_{\alpha}\right) \cos \left(\underline{k}_{\alpha}(a-b)\right) \\
& -\left(\underline{k}_{\alpha}^{2} \underline{B}_{\alpha} \underline{D}_{\alpha}\right. \\
& \left.+\frac{\bar{A}_{\alpha} \bar{C}_{\alpha}}{\underline{k}_{\alpha}}\right) \sin \left(\underline{k}_{\alpha}(a-b)\right), \\
W\left(\bar{\varphi}_{\alpha}, \bar{\chi}_{\alpha}\right)(\lambda)= & \left(\frac{\underline{A}_{\alpha} \bar{D}_{\alpha}}{\bar{k}_{\alpha}}\right. \\
& \left.-\bar{k}_{\alpha}^{2} \bar{B}_{\alpha} \underline{C}_{\alpha}\right) \cos \left(\bar{k}_{\alpha}(a-b)\right) \\
& -\left(\bar{k}_{\alpha}^{2} \bar{B}_{\alpha} \bar{D}_{\alpha}\right. \\
& \left.\underline{A}_{\alpha} \underline{C}_{\alpha}\right) \sin \left(\underline{k}_{\alpha}(a-b)\right) .
\end{aligned}
$$

Example 1. Consider the fuzzy eigenvalues and fuzzy eigenfunctions of the problem

$$
\begin{gathered}
u^{\prime \prime}+[\lambda]^{\alpha} u=0, t \in(0,1) \\
-u(0)+[\lambda]^{\alpha}[2]^{\alpha} u^{\prime}(0)=0, \\
{[-1]^{\alpha} u(1)+u^{\prime}(1)=0,}
\end{gathered}
$$

where $[A]^{\alpha}=-1,[B]^{\alpha}=[2]^{\alpha}=[1+\alpha, 3-\alpha]$, $[C]^{\alpha}=[-1]^{\alpha}=[-2+\alpha,-\alpha],[D]^{\alpha}=1$ and $[\lambda]^{\alpha}=\left[\underline{\lambda}_{\alpha}, \bar{\lambda}_{\alpha}\right]$ positive fuzzy eigenvalue parameter and $u(t, \lambda)$ is positive fuzzy function.

Let be $[\lambda]^{\alpha}=\left[\underline{\lambda}_{\alpha}, \bar{\lambda}_{\alpha}\right]=\left[\underline{k}_{\alpha}^{2}, \bar{k}_{\alpha}^{2}\right], \underline{k}_{\alpha}>0$, $\bar{k}_{\alpha}>0$. Solution of the equation (19) satisfying the conditions (20) is

$$
\begin{gathered}
\underline{\varphi}_{\alpha}(t, \lambda)=\underline{k}_{\alpha}^{2}(1+\alpha) \cos \left(\underline{k}_{\alpha} t\right)+\frac{1}{\underline{k}_{\alpha}} \sin \left(\underline{k}_{\alpha} t\right), \\
\bar{\varphi}_{\alpha}(t, \lambda)=\bar{k}_{\alpha}^{2}(3-\alpha) \cos \left(\bar{k}_{\alpha} t\right)+\frac{1}{\bar{k}_{\alpha}} \sin \left(\bar{k}_{\alpha} t\right), \\
{[\varphi(t, \lambda)]^{\alpha}=\left[\underline{\varphi}_{\alpha}(t, \lambda), \bar{\varphi}_{\alpha}(t, \lambda)\right]}
\end{gathered}
$$

and solution of the equation (19) satisfying the conditions (21) is

$$
\begin{aligned}
& \underline{\chi}_{\alpha}(t, \lambda)=\left(\frac{1}{\underline{k}_{\alpha}} \cos \left(\underline{k}_{\alpha}\right)\right. \\
& \left.-\frac{\alpha}{\underline{k}_{\alpha}} \sin \left(\underline{k}_{\alpha}\right)\right) \cos \left(\underline{k}_{\alpha} t\right) \\
& +\left(\frac{1}{\underline{k}_{\alpha}} \sin \left(\underline{k}_{\alpha}\right)\right. \\
& \left.+\frac{\alpha}{\underline{k}_{\alpha}} \cos \left(\underline{k}_{\alpha}\right)\right) \sin \left(\underline{k}_{\alpha} t\right), \\
& \bar{\chi}_{\alpha}(t, \lambda)=\left(\frac{1}{\bar{k}_{\alpha}} \cos \left(\bar{k}_{\alpha}\right)\right. \\
& \left.-\frac{(2-\alpha)}{\bar{k}_{\alpha}} \sin \left(\bar{k}_{\alpha}\right)\right) \cos \left(\bar{k}_{\alpha} t\right) \\
& +\left(\frac{1}{\bar{k}_{\alpha}} \sin \left(\bar{k}_{\alpha}\right)\right. \\
& \left.+\frac{(2-\alpha)}{\bar{k}_{\alpha}} \cos \left(\bar{k}_{\alpha}\right)\right) \sin \left(\bar{k}_{\alpha} t\right), \\
& {[\chi(t, \lambda)]^{\alpha}=\left[\underline{\chi}_{\alpha}(t, \lambda), \bar{\chi}_{\alpha}(t, \lambda)\right] .} \\
& W\left(\underline{\varphi}_{\alpha}, \underline{\chi}_{\alpha}\right)(\lambda)=\left(\underline{k}_{\alpha}^{2} \alpha(1+\alpha)\right. \\
& \left.-\frac{1}{\underline{k}_{\alpha}}\right) \cos \left(\underline{k}_{\alpha}\right) \\
& +\left(\underline{k}_{\alpha}^{2}(1+\alpha)\right. \\
& \left.+\frac{\alpha}{\underline{k}_{\alpha}}\right) \sin \left(\underline{k}_{\alpha}\right), \\
& W\left(\bar{\varphi}_{\alpha}, \bar{\chi}_{\alpha}\right)(\lambda)=\left(\bar{k}_{\alpha}^{2}(2-\alpha)(3-\alpha)\right. \\
& \left.-\frac{1}{\bar{k}_{\alpha}}\right) \cos \left(\bar{k}_{\alpha}\right) \\
& +\left(\bar{k}_{\alpha}^{2}(3-\alpha)\right. \\
& \left.+\frac{(2-\alpha)}{\bar{k}_{\alpha}}\right) \sin \left(\bar{k}_{\alpha}\right) \text {. }
\end{aligned}
$$

Since the eigenvalues of the fuzzy boundary value problem (19)- (21) if and only if are consist of the zeros of functions $\underline{W}_{\alpha}(\lambda)=W\left(\underline{\varphi}_{\alpha}, \underline{\chi}_{\alpha}\right)(\lambda)$ and $\bar{W}_{\alpha}(\lambda)=W\left(\bar{\varphi}_{\alpha}, \bar{\chi}_{\alpha}\right)(\lambda)$, computing the values $\underline{k}_{\alpha}$ satisfying the equation $\underline{W}_{\alpha}(\lambda)=0$ and $\bar{k}_{\alpha}$ satisfying the equation $\bar{W}_{\alpha} \overline{ }_{(\lambda)}=0$ for each $\alpha \in[0,1]$, we get infinitely many values as

$$
\alpha=0 \Rightarrow \quad \begin{array}{cc}
\underline{k}_{1}=0.915811, & \bar{k}_{1}=0.343085, \\
\underline{k}_{2}=3.17289, & \bar{k}_{2}=2.0719, \\
\underline{k}_{3}=6.28721, & \bar{k}_{3}=5.17844,
\end{array}
$$




$$
\begin{aligned}
& \underline{k}_{1}=0.808395, \quad \bar{k}_{1}=0.368214,
\end{aligned}
$$

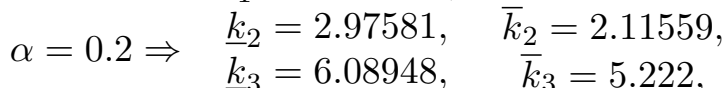

$$
\begin{aligned}
& \begin{array}{rlrl}
\underline{k}_{1} & =0.674971, & \bar{k}_{1}=0.413302, \\
\alpha=0.5 \Rightarrow & \underline{k}_{2}=2.71138, & \bar{k}_{2}=2.19653, \\
\underline{k}_{3}=5.82291, & \bar{k}_{3}=5.30307,
\end{array} \\
& \alpha=0.8 \Rightarrow \begin{array}{cc}
\underline{k}_{1}=0.571662, & \bar{k}_{1}=0.470075, \\
\underline{k}_{2}=2.50229, & \bar{k}_{2}=2.30274, \\
\underline{k}_{3}=5.61159, & \bar{k}_{3}=5.41,
\end{array} \\
& \underline{k}_{1}=0.516499, \quad \bar{k}_{1}=0.516499, \\
& \alpha=1 \Rightarrow \quad \begin{array}{cl}
\underline{k}_{2}=2.39268, & \bar{k}_{2}=2.39268, \\
\underline{k}_{3}=5.50079, & \bar{k}_{3}=5.50079,
\end{array}
\end{aligned}
$$

We show that this values are $\underline{k}_{n}$ and $\bar{k}_{n}, k=1,2, \ldots$ for each $\alpha \in[0,1]$. Then, the eigenvalues are $\left[\lambda_{n}\right]^{\alpha}=\left[\underline{\lambda}_{\alpha, n}, \bar{\lambda}_{\alpha, n}\right]=\left[\underline{k}_{\alpha, n}^{2}, \bar{k}_{\alpha, n}^{2}\right]$ with associated solutions

$$
\begin{aligned}
{\left[\varphi_{n}(t, \lambda)\right]^{\alpha}=} & {\left[\underline{\varphi}_{\alpha, n}(t, \lambda), \bar{\varphi}_{\alpha, n}(t, \lambda)\right], } \\
\underline{\varphi}_{\alpha, n}(t, \lambda)= & \underline{k}_{\alpha, n}^{2}(1+\alpha) \cos \left(\underline{k}_{\alpha, n} t\right) \\
& +\frac{1}{\underline{k}_{\alpha, n}} \sin \left(\underline{k}_{\alpha, n} t\right), \\
\bar{\varphi}_{\alpha, n}(t, \lambda)= & \bar{k}_{\alpha, n}^{2}(3-\alpha) \cos \left(\bar{k}_{\alpha, n} t\right) \\
& +\frac{1}{\underline{k}_{\alpha, n}} \sin \left(\bar{k}_{\alpha, n} t\right)
\end{aligned}
$$

and

$$
\begin{aligned}
{\left[\chi_{n}(t, \lambda)\right]^{\alpha}=} & {\left[\underline{\chi}_{\alpha, n}(t, \lambda), \bar{\chi}_{\alpha, n}(t, \lambda)\right], } \\
\underline{\chi}_{\alpha, n}(t, \lambda)= & \left(\frac{1}{\underline{k}_{\alpha, n}} \cos \left(\underline{k}_{\alpha, n}\right)\right. \\
& \left.-\frac{\alpha}{\underline{k}_{\alpha, n}} \sin \left(\underline{k}_{\alpha, n}\right)\right) \cos \left(\underline{k}_{\alpha, n} t\right) \\
& +\left(\frac{1}{\underline{k}_{\alpha, n}} \sin \left(\underline{k}_{\alpha, n}\right)\right. \\
& \left.+\frac{\alpha}{\underline{k}_{\alpha, n}} \cos \left(\underline{k}_{\alpha, n}\right)\right) \sin \left(\underline{k}_{\alpha, n} t\right),
\end{aligned}
$$

$$
\begin{aligned}
\bar{\chi}_{\alpha, n}(t, \lambda)= & \left(\frac{1}{\bar{k}_{\alpha, n}} \cos \left(\bar{k}_{\alpha, n}\right)\right. \\
& \left.-\frac{(2-\alpha)}{\bar{k}_{\alpha, n}} \sin \left(\bar{k}_{\alpha, n}\right)\right) \cos \left(\bar{k}_{\alpha, n} t\right) \\
& +\left(\frac{1}{\bar{k}_{\alpha, n}} \sin \left(\bar{k}_{\alpha, n}\right)\right. \\
& \left.+\frac{(2-\alpha)}{\bar{k}_{\alpha, n}} \cos \left(\bar{k}_{\alpha, n}\right)\right) \sin \left(\bar{k}_{\alpha, n} t\right) .
\end{aligned}
$$

When

$$
\begin{aligned}
\frac{\partial \underline{\varphi}_{\alpha, n}(t, \lambda)}{\partial \alpha} & \geq 0, \frac{\partial \bar{\varphi}_{\alpha, n}(t, \lambda)}{\partial \alpha} \leq 0 \\
\underline{\varphi}_{\alpha, n}(t, \lambda) & \leq \bar{\varphi}_{\alpha, n}(t, \lambda)
\end{aligned}
$$

$$
\begin{aligned}
\frac{\partial \underline{\chi}_{n, \alpha}(t, \lambda)}{\partial \alpha} & \geq 0, \frac{\partial \bar{\chi}_{n, \alpha}(t, \lambda)}{\partial \alpha} \leq 0 \\
\underline{\chi}_{n, \alpha}(t, \lambda) & \leq \bar{\chi}_{n, \alpha}(t, \lambda)
\end{aligned}
$$

for all $n=1,2, \ldots,\left[\varphi_{n}(t, \lambda)\right]^{\alpha}$ and $\left[\chi_{n}(t, \lambda)\right]^{\alpha}$ are valid $\alpha$-level sets. That is, $\left[\varphi_{n}(t, \lambda)\right]^{\alpha}$ and $\left[\chi_{n}(t, \lambda)\right]^{\alpha}$ are eigenfunctions when (22) and (23) are satisfied.

Now, we draw the graphics of $\left[\varphi_{n}(t, \lambda)\right]^{\alpha}$ and $\left[\chi_{n}(t, \lambda)\right]^{\alpha}$ for $\alpha=0.2$ and $n=2$.

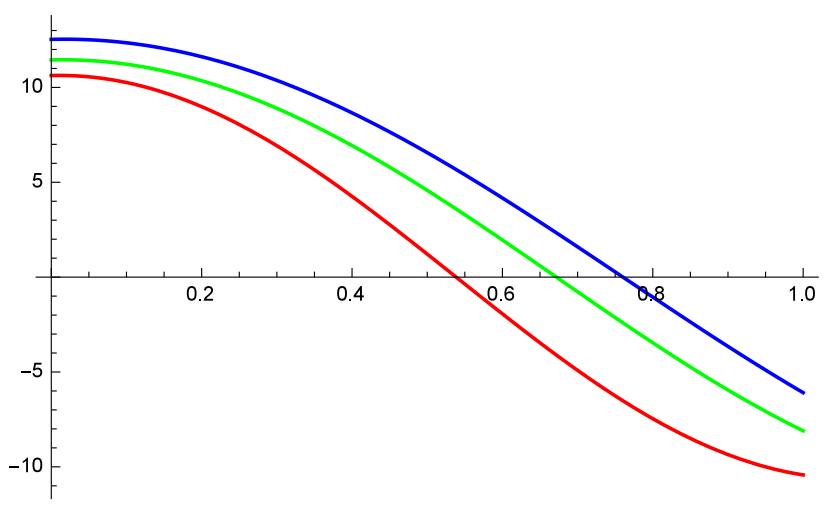

Figure 1. Graphic of $\left[\varphi_{n}(t, \lambda)\right]^{\alpha}$ : Red $\rightarrow \quad \underline{\varphi}_{\alpha, n}(t, \lambda), \quad$ Blue $\rightarrow \bar{\varphi}_{\alpha, n}(t, \lambda), \quad$ Green $\rightarrow \underline{\varphi}_{1, n}(t, \lambda)=$ $\bar{\varphi}_{1, n}(t, \lambda)$. 


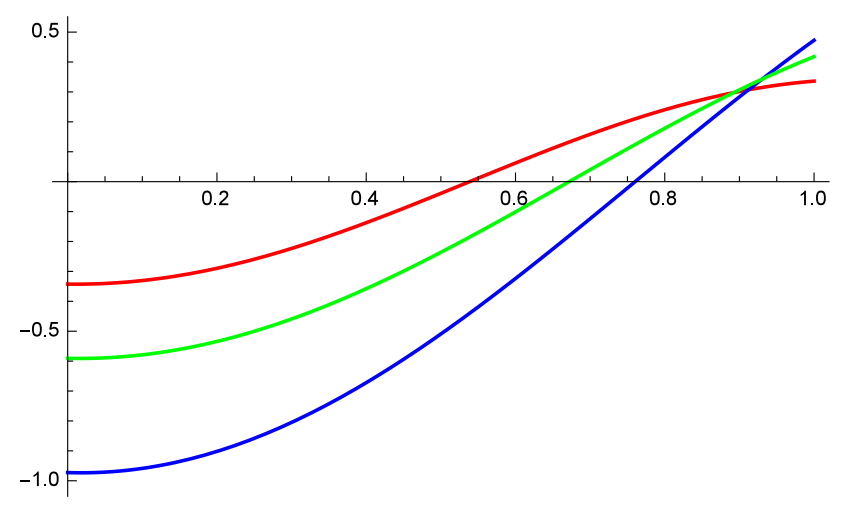

Figure 2. Graphic of $\left[\chi_{n}(t, \lambda)\right]^{\alpha}$ : Red $\rightarrow \quad \underline{\chi}_{\alpha, n}(t, \lambda), \quad$ Blue $\rightarrow \bar{\chi}_{\alpha, n}(t, \lambda)$, Green $\rightarrow \underline{\chi}_{1, n}(t, \lambda)=$ $\bar{\chi}_{1, n}(t, \lambda)$.

In Figure $1,\left[\varphi_{n}(t, \lambda)\right]^{\alpha}$ is a valid $\alpha$-level set for $t \in[0,0.538478]$ and in Figure 2, is a valid $\alpha$-level set for $t \in[0.912106,1]$, since the inequalities (23) and the solution is positive fuzzy function.

Then, the eigenfunctions are $\left[\varphi_{n}(t, \lambda)\right]^{\alpha}$ on $[0,0.538478]$ and $\left[\chi_{n}(t, \lambda)\right]^{\alpha}$ on $[0.912106,1]$ associated with eigenvalues $\left[\lambda_{n}\right]^{\alpha}=\left[\underline{\lambda}_{\alpha, n}, \bar{\lambda}_{\alpha, n}\right]=$ $\left[\underline{k}_{\alpha, n}^{2}, \bar{k}_{\alpha, n}^{2}\right]$ for $\alpha=0.2$ and $n=2$.

\section{Conclusion}

In this work, we study the problem with fuzzy eigenvalue parameter in one of the boundary conditions. We find infinitely many eigenvalues for each $\alpha \in[0,1]$. Also, we find solutions associated with eigenvalues. We draw graphics of solutions. But solutions are not valid $\alpha$-level sets every time. That is, solutions are valid fuzzy functions different interval for each $\alpha \in[0,1]$. Thus, found solutions are solutions only in interval which they are valid fuzzy function. That is, found solutions are eigenfunctions only in interval which they are valid fuzzy function.

\section{References}

[1] Irkin, R., Yılmaz Özgür, N., Taş, N. (2018). Optimization of lactic acid bacteria viability using fuzzy soft set modelling. An International Journal of Optimization and Control: Theories \& Applications, 8(2), 266-275.

[2] Karakaş, E., Özpalamutçu, H. (2019). A genetic algorithm for fuzzy order acceptance and scheduling problem. An International Journal of Optimization and Control: Theories \& Applications, 9(2), 186-196.

[3] Fulton, C. T. (1977). Two point boundary value problems with eigenvalue parameter contained in the boundary conditions. Proc. Soc. Edinburg, 77A, 293-308.

[4] Binding, P. A., Browne, P. J., Watson, B. A. (2002). Sturm-Liouville problems with boundary conditions rationally dependent on the eigenparameter, II. Journal of Computational and Applied Mathematics, 148(1), 147168.

[5] Gültekin Çitil, H., Altınışık, N. (2017). On the eigenvalues and the eigenfunctions of the Sturm-Liouville fuzzy boundary value problem. Journal of Mathematical and Computational Science, 7(4), 786-805.

[6] Gültekin Çitil, H., Altınışık, N. (2018). The examination of eigenvalues and eigenfunctions of the Sturm-Liouville fuzzy problem according to boundary conditions. International Journal of Mathematical Combinatorics, 1, 51-60.

[7] Gültekin Çitil, H., Altınışık, N. (2018). The eigenvalues and the eigenfunctions of the Sturm-Liouville fuzzy problem with fuzzy coefficient boundary conditions. Journal of Science and Arts, 4(45), 947-958.

[8] Gültekin Çitil, H. (2017). The eigenvalues and the eigenfunctions of the SturmLiouville fuzzy boundary value problem according to the generalized differentiability. Scholars Journal of Physics, Mathematics and Statistics, 4(4), 185-195.

[9] Ceylan, T., Altınışık, N. (2018). Eigenvalue problem with fuzzy coefficients of boundary conditions. Scholars Journal of Physics, Mathematics and Statistics, 5(2), 187-193.

[10] Gültekin Çitil, H. (2019). Important notes for a fuzzy boundary value problem. Applied Mathematics and Nonlinear Sciences, 4(2), 305-314.

[11] Ceylan, T., Altınışık, N. (2018). Fuzzy eigenvalue problem with eigenvalue parameter contained in the boundary condition. Journal of Science and Arts, 3(44), 589-602.

[12] Gültekin Çitil, H. (2019). Sturm-Liouville fuzzy problem with fuzzy eigenvalue parameter. International Journal of Mathematical Modelling \& Computations, 9(3), 187- 195.

[13] Liu, H.-K. (2011). Comparison results of twopoint fuzzy boundary value problems. International Journal of Computational and Mathematical Sciences, 5(1), 1-7.

[14] Khastan, A., Nieto, J. J. (2010). A boundary value problem for second order fuzzy differential equations. Nonlinear Analysis, 72, 35833593. 
[15] Shirin, S., Saha, G. K. (2011). A new computational methodology to find appropriate solutions of fuzzy equations. Mathematical Theory and Modeling, 2(1), 1-10.

[16] Lakshmikantham, V., Mohapatra, R. N. (2003). Theory of Fuzzy Differential Equations and Inclusions. Taylor and Francis, London, New York.

[17] Puri, M. L., Ralescu D. A. (1983). Differentials of fuzzy functions. Journal of Mathematical Analysis and Applications, 91(2), 552558.

[18] Bede, B. (2008). Note on "Numerical solutions of fuzzy differential equations by predictor-corrector method". Information Sciences, 178(7), 1917-1922.

Hülya Gültekin Citil is an Assistant Prof. Dr. at the Department of Mathematics, Faculty of Arts and Sciences, Giresun University, Turkey. She received her B.Sc. (2007) degree from Department of Mathematics, Faculty of Arts and Sciences, Ondokuz Mayıs University and M.Sc. (2010) degree and Ph.D. (2015) degree from Ondokuz Mayıs University, Turkey. She has many research papers about the fuzzy initial and boundary value problems and Sturm-Liouville fuzzy problems.

An International Journal of Optimization and Control: Theories \& Applications (http://ijocta.balikesir.edu.tr)

This work is licensed under a Creative Commons Attribution 4.0 International License. The authors retain ownership of the copyright for their article, but they allow anyone to download, reuse, reprint, modify, distribute, and/or copy articles in IJOCTA, so long as the original authors and source are credited. To see the complete license contents, please visit http://creativecommons.org/licenses/by/4.0/. 